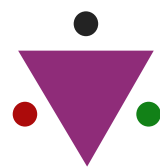

IJCRR

Section: Healthcare

Sci. Journal

Impact Factor

4.016

ICV: 71.54

\title{
Fine Needle Aspiration Cytology
}

FNAC (Fine Needle Aspiration Cytology) is a simple, inexpensive, easily performed outpatient procedure which can provide a rapid diagnosis. It has been widely used in Europe for decades, mainly in Scandinavian countries.

A technique which is safe, rapid, relatively pain free, cost effective \& accurate is always a clinician's first choice and this is what FNAC is about.

It is eminently suitable as first line investigation for almost all superficial palpable swellings as well as many deep seated lesions. FNAC was initially conceived as a means to confirm a clinical suspicion of local recurrence or metastasis of known cancer without subjecting the patient to further surgical intervention.

FNAC is a time tested simple office procedure having a high degree of diagnostic accuracy \& precision. The specificity \& sensitivity of diagnostic precision lie in range of $60 \%$ \& $80 \%$ respectively.

The acceptance both by surgeons \& pathologists itself speaks of the tale of comfort which it allows.

The art of medicine is practiced within a community of caregivers who are perched on innumerable speciality branches and these braches intersect each other at various times.

Clinical consultations help to acquaint cytopathologist about probable diagnoses possible for any lesion. Often a major surgical biopsy can be avoided by performing a needle aspiration instead.

Surgeons are always impressed by the help of FNAC to make diagnoses which affect treatment of patient in a wide manner. Many tumours being diagnosed high grade on FNAC make the surgeon to go for chemotherapy before surgical intervention.

Conclusion - Fine needle aspiration cytology has an utmost importance in the current era of surgical practice in the preoperative stage as it guides the clinician a lot in the treatment plan and mostly clear the pathological aspects of the disease avoid untoward complications related to disease and treatment for the sake of pathological diagnosis. Many times it avoids unnecessary surgical intervention.

Wishing the readers a happy reading of this new issue

Corresponding Author:

Dr. Sachin B. Ingle [MD Pathology], Professor \& Surgical Pathologist, Department of Pathology, MIMSR Medical College, Shri Siddheshwar Surgical Pathology \& Research Centre, Latur 413512, Maharshtra India; "Editor in chief "International Journal of Current Research \& Reviews", India; Email: dr.sachiningle@gmail.com.

ISSN: 2231-2196 (Print)

ISSN: 0975-5241 (Online) 